\title{
Diffusion in two-component quasiparticle systems of liquid and solid mixtures of helium isotopes
}

\author{
I.N.Adamenko ${ }^{1}$, K.E.Nemchenko ${ }^{1}$, A.V.Zhukov ${ }^{1}$, \\ Thomas F. George ${ }^{2}$, Lakshmi N. Pandey ${ }^{3}$, Chung-In Um ${ }^{4}$ \\ 1 Department of Physics and Technology, Kharkiv State University, \\ 4 Svobody Sq., Kharkiv, 310077, Ukraine \\ 2 Office of Chancellor, Departments of Chemistry, Physics and Astronomy, \\ University of Wisconsin-Stevens Point, Wisconsin 54481-3897, U.S.A. \\ 3 Departments of Chemistry and Physics, Washington State University, \\ Pullman, Washington 99164-4630, U.S.A. \\ 4 Department of Physics, College of Science, Korea University, \\ Seoul 136-701, Korea
}

\section{Received December 5, 1997}

An exact expression for the diffusion time which depends on the interaction rates for particles of not only different, but also of the same species, has been derived from the system of kinetic equations. The result is valid for particles with arbitrary statistics and energy-momentum relations. The derived general relations are valid for investigating diffusion in liquid and solid ${ }^{3} \mathrm{He}-{ }^{4} \mathrm{He}$ mixtures. The contribution of interaction between quasiparticles of the same type to the diffusion coefficient and effective thermal conductivity of superfluid solutions is analyzed. The calculated values are compared with experimental data. The calculated diffusion coefficient of ${ }^{3} \mathrm{He}-{ }^{4} \mathrm{He}$ solid solutions differs from the previous theoretical results. A comparison of the obtained diffusion coefficient with experimental data makes it possible to determine the numerical value of the energy band width for impurity quasiparticles.

Key words: diffusion, thermal conductivity, helium, quantum liquid, quantum solid, superfluids, ${ }^{3} \mathrm{He}-{ }^{4} \mathrm{He}$ mixtures, phonons, rotons, impuritons.

PACS: $51.20 .+d, 67.80 . \mathrm{Mg}$

\section{Introduction}

Investigation of diffusion processes in condensed media, in which a quasiparticle description is valid, is one of the most important problems of modern classical 
and quantum kinetics. The present paper deals with the diffusion of impurity excitations in weakly-concentrated solutions of ${ }^{3} \mathrm{He}$ in superfluid and solid ${ }^{4} \mathrm{He}$. The general result obtained for a two-component gaseous system with arbitrary statistics and the dispersion law makes it possible to describe the quasiparticle systems in some limiting cases of interest.

For every classical and quantum two-component gaseous system known to us, the diffusion coefficients can be written in the form

$$
D=u_{\mathrm{D}}^{2} \tau_{\mathrm{D}}
$$

where $u_{\mathrm{D}}$ is a typical velocity, whose analytical expression is determined by the dispersion law and statistics of the particles, and $\tau_{\mathrm{D}}$ is a typical diffusion time. The solution of a set of two linearized kinetic equations for a mixture of $\alpha$-type and $\beta$-type gases with any dispersion law and chemical potential takes the form $($ see $[1])$

$$
\tau_{\mathrm{D}}=-\left\langle\varphi_{\mathrm{D}}\left|(\hat{S}+\hat{I})^{-1}\right| \varphi_{\mathrm{D}}\right\rangle
$$

where $\hat{S}$ and $\hat{I}$ are the matrices in the $2 \mathrm{D}$ space of component momenta. The matrix

$$
\hat{S}=\left(\begin{array}{cc}
J_{\alpha \alpha} & 0 \\
0 & J_{\beta \beta}
\end{array}\right)
$$

includes the operators of collisions between particles of the same type, and the matrix

$$
\hat{I}=\left(\begin{array}{cc}
J_{\alpha \beta} & J_{\alpha \beta} \\
J_{\beta \alpha} & J_{\beta \alpha}
\end{array}\right)
$$

consists of the operators of collisions between particles of different types. The collision operators $J_{k l}(k, l=\alpha, \beta)$ are linearized integrals of the collision of $k$-type particles with $l$-type particles and in a usual way can be expressed by the transition probability density function.

\section{Diffusion time}

The time $\tau_{\mathrm{D}}$ can be expressed as a scalar product determined in the following way:

$$
\langle\psi \mid \chi\rangle=\sum_{k=\alpha, \beta}{ }_{1}\left\langle\psi_{k} \mid \chi_{k}\right\rangle_{1}=-\sum_{k=\alpha, \beta} \int \psi_{k}^{*} \chi_{k} f_{0 k}^{\prime} \mathrm{d} \Gamma_{k} .
$$

Here and below, the subscript 1 on a bra- or a ket-vector denotes a $1 \mathrm{D}$ vector, and $f_{0 k}^{\prime}$ is a derivative of the local equilibrium distribution function with respect to energy. The vector $\left|\varphi_{\mathrm{D}}\right\rangle$ that determines $\tau_{\mathrm{D}}$ is normalized with respect to the scalar product (5) as

$$
\left|\varphi_{\mathrm{D}}\right\rangle=\left(\rho \rho_{\alpha} \rho_{\beta}\right)^{-\frac{1}{2}}\left|\begin{array}{c}
\rho_{\beta} p_{\alpha z} \\
-\rho_{\alpha} p_{\beta z}
\end{array}\right\rangle
$$


where $p_{k z}$ is the $z$-th component of the momentum $(k=\alpha, \beta)$, and

$$
\rho_{k}={ }_{1}\left\langle p_{k z} \mid p_{k z}\right\rangle_{1}
$$

is the normal density of the $k$-th component, and $\rho=\rho_{\alpha}+\rho_{\beta}$ is the total density of the mixture.

To obtain an exact expression for the matrix elements (2), one should introduce a complete set of orthonormal two-component vectors $\left|\varphi_{n}\right\rangle(n=1,2, .$.$) . The first$ vector of this set corresponds to the total momentum of the two-component system of quasiparticles,

$$
\left|\varphi_{1}\right\rangle=\rho^{-1}\left|\begin{array}{l}
p_{\alpha z} \\
p_{\beta z}
\end{array}\right\rangle
$$

and the second one should be taken as

$$
\left|\varphi_{2}\right\rangle=\left|\varphi_{\mathrm{D}}\right\rangle
$$

The remaining vectors can be constructed by using the standard procedure (see, e.g., [2]) and the definition of the scalar product (5). By constructing a complete set of vectors in this way, the expression for $\tau_{\mathrm{D}}$

$$
\tau_{\mathrm{D}}=-\left\{[\hat{S}+\hat{I}]^{-1}\right\}_{22}
$$

can be rewritten as

$$
\tau_{\mathrm{D}}=-\left\{I_{22}-\sum_{n, n^{\prime}=3}^{\infty} I_{2 n}\left[(\tilde{I}+\tilde{S})^{-1}\right]_{n n^{\prime}} I_{n^{\prime} 2}\right\}^{-1} .
$$

Here the square matrices $\tilde{I}$ and $\tilde{S}$ include the matrix elements

$$
(\tilde{I})_{n n^{\prime}}=I_{n n^{\prime}}, \quad(\tilde{S})_{n n^{\prime}}=S_{n n^{\prime}}
$$

where

$$
I_{n n^{\prime}}=\left\langle\varphi_{n}|\hat{I}| \varphi_{n}^{\prime}\right\rangle, \quad S_{n n^{\prime}}=\left\langle\varphi_{n}|\hat{S}| \varphi_{n}^{\prime}\right\rangle .
$$

The matrices $\tilde{I}$ and $\tilde{S}$ are infinite and nondiagonal. Therefore, the exact solution (11) does not allow us to obtain an explicit analytical expression for $\tau_{\mathrm{D}}$. However, the solution (11) makes it possible to consider various limiting cases, to find minimal and maximal values of $\tau_{\mathrm{D}}$, to obtain correct interpolation formulae and to carry out computer calculations for various physical systems.

So, in the case of fast equilibrium established between particles of the same type, i.e. when the inequality

$$
S_{n n^{\prime}} \gg I_{n n^{\prime}}
$$

is valid, one can obtain from equation (11)

$$
\tau_{\mathrm{D}}=\tau_{\mathrm{D} \min }=-\frac{1}{I_{22}}=\left(\tau_{\alpha \beta}^{(0)^{-1}}+\tau_{\beta \alpha}^{(0)^{-1}}\right)^{-1},
$$


where

$$
\tau_{k l}^{(0)^{-1}}=-\left\langle J_{k l}\right\rangle_{k} ; \quad k, l=\alpha, \beta ; \quad k \neq l .
$$

Here and below, the normalized average of the arbitrary operator $\hat{\mathcal{L}}$ with respect to the quasiparticle momentum is denoted as

$$
\langle\hat{\mathcal{L}}\rangle_{k}=\rho_{k}^{-1}\left\langle p_{k z}|\hat{\mathcal{L}}| p_{k z}\right\rangle_{1}
$$

According to the momentum conservation law and definition (16),

$$
\tau_{\alpha \beta}^{(0)^{-1}}=\frac{\rho_{\beta}}{\rho_{\alpha}} \tau_{\beta \alpha}^{(0)}{ }^{-1}
$$

Using the fact that the operators $\tilde{I}$ and $\tilde{S}$ are Hermitian and defined as negative, it can be shown that

$$
\tau_{\mathrm{D}} \geqslant \tau_{\mathrm{Dmin}}
$$

In the opposite limiting case of the slow establishing of equilibrium between identical particles $\left(S_{\beta \beta} \ll I_{\beta \beta \prime}\right), \tau_{\mathrm{D}}$ reaches its maximum value

$$
\tau_{\mathrm{D} \max }=\left\langle\varphi_{2}\left|\hat{I}^{-1}\right| \varphi_{2}\right\rangle .
$$

When the density of the $\alpha$-component is relatively low $\left(\rho_{\alpha} \ll \rho_{\beta}\right)$ and the relaxation time in the $\alpha$-component is great $\left(J_{\alpha \alpha} \rightarrow 0\right)$, from (11) we obtain

$$
\tau_{\mathrm{D}}=\tau_{\alpha \beta}^{(\infty)},
$$

where

$$
\tau_{\alpha \beta}^{(\infty)}=-\left\langle J_{\alpha \beta}^{-1}\right\rangle_{\alpha}
$$

It should be noted that (16) is the average of the rate, but (22) is the average of the time, and one can prove that for arbitrary momentum dependence of $J_{\alpha \beta}$, the time (22) is greater than the time defined by (16).

For a definite physical system, the rate $\tau_{\alpha \beta}^{(0)^{-1}}$ can be easily calculated, but to calculate $\tau_{\alpha \beta}^{(\infty)^{-1}}(22)$ one must find the inverse operator to the integral operator $J_{\alpha \beta}$, which can be done only by using some approximations. The exact expression (11) and the limiting formulae (15), (20) and (22) make it possible to propose the correct relaxation-time approximation for a two-component system:

$$
\begin{gathered}
\hat{S}=\left(\begin{array}{cc}
-t_{\alpha \alpha}^{-1}+t_{\alpha \alpha}^{-1}\left|p_{\alpha z}\right\rangle_{1} \frac{\tau_{\alpha \alpha}^{(0)}}{\rho_{\alpha}}{ }_{1}\left\langle p_{\alpha z}\right| t_{\alpha \alpha}^{-1} & 0 \\
0 & -t_{\beta \beta}^{-1}+t_{\beta \beta}^{-1}\left|p_{\beta z}\right\rangle_{1}{ }_{\frac{\tau_{\beta \beta}}{\rho_{\beta}}{ }_{1}\left\langle p_{\beta z}\right| t_{\beta \beta}^{-1}}
\end{array}\right), \\
\hat{I}=\left(\begin{array}{cc}
-t_{\alpha \beta}^{-1} & t_{\alpha \beta}^{-1}\left|p_{\alpha z}\right\rangle_{1} \rho_{\alpha}^{-1} \tau_{\alpha \beta 1}^{(0)}\left\langle p_{\beta z}\right| t_{\beta \alpha}^{-1} \\
t_{\beta \alpha}^{-1}\left|p_{\beta z}\right\rangle_{1} \rho_{\beta}^{-1} \tau_{\beta \alpha 1}^{(0)}{ }_{1}\left\langle p_{\alpha z}\right| t_{\alpha \beta}^{-1} & -t_{\beta \alpha}^{-1}
\end{array}\right) .
\end{gathered}
$$


This approximation satisfies the conservation of the $z$-th component of the total momentum of the system:

$$
\hat{I}\left|\varphi_{1}\right\rangle=0
$$

Here

$$
t_{k l}^{-1}\left(p_{k}\right)=\frac{\nu_{k l}\left(p_{k}\right)}{\left\langle\nu_{k l}\left(p_{k}\right)\right\rangle_{k}} \tau_{k l}^{(0)^{-1}} \quad(k \neq l)
$$

and $\nu_{k l}$ is a transport scattering rate that is determined in a usual way from collision integrals.

The model (23) can be used for the calculation of $\tau_{\mathrm{D}}$ to give

$$
\tau_{\mathrm{D}}=\tau_{\mathrm{D} \min }+\frac{\rho_{\beta}}{\rho}\left(\tau_{\alpha \beta}-\tau_{\alpha \beta}^{(0)}\right)+\frac{\rho_{\alpha}}{\rho}\left(\tau_{\beta \alpha}-\tau_{\beta \alpha}^{(0)}\right),
$$

where

$$
\begin{gathered}
\tau_{k l}=\left\langle R_{k}\right\rangle+\left\langle R_{k} t_{k k}^{-1}\right\rangle_{k}^{2}\left\langle R_{k} t_{k k}^{-1} t_{k l}^{-1}\right\rangle_{k}^{-1}, \\
R_{k}=\left(t_{k k}^{-1}+t_{k l}^{-1}\right)^{-1} .
\end{gathered}
$$

The time $\tau_{k l}$ in (26) depends on the relaxation rates $t_{k k}^{-1}$ of the $k$-particles. Note, the expression (26) obtained in the limiting cases gives not only the formulae (16), (20) and (22) of this paper, but also the results of other theoretical investigations [3] and, in particular, the well-known Callaway formula [4].

\section{Thermal conductivity of quasiparticle systems of dilute so- lutions of ${ }^{3} \mathrm{He}$ in superfluid ${ }^{4} \mathrm{He}$}

Because of the nature of thermal excitations, diffusion in such mixtures defines thermal conductivity of the matter that includes these thermal excitations [5]. Consider a superfluid mixture of helium isotopes. The kinetic properties of the mixtures are determined by a set of quasiparticles: phonons and rotons (thermal excitations of He II) and impuritons ( ${ }^{3} \mathrm{He}$ quasiparticles). It is useful to separate three temperature regions which differ one from another by the types of physical processes which govern the thermal conductivity of the mixture.

At low temperatures $(T<0.6 \mathrm{~K})$, when the roton contribution can be neglected, the thermal conductivity of the mixtures is determined by diffusion in the phonon-impuriton system:

$$
\kappa_{\mathrm{eff}}=D_{\mathrm{iph}}\left(S_{\mathrm{ph}} \frac{\rho_{\mathrm{ph}}+\rho_{i}}{\rho_{\mathrm{ph}}}\right)^{2} \frac{1}{n_{3}}+\kappa_{3} .
$$

Here

$$
D_{\mathrm{iph}}=\frac{4}{9} \frac{{ }_{1}\left\langle\varepsilon_{\mathrm{i}} \mid 1\right\rangle_{1}^{2}}{{ }_{1}\langle 1 \mid 1\rangle_{1}} \frac{\rho_{\mathrm{ph}}}{\rho_{\mathrm{ph}}+\rho_{\mathrm{i}}} \frac{1}{\rho_{\mathrm{i}}} \tau_{\mathrm{D}}^{(\mathrm{iph})}
$$

is a coefficient of diffusion of impuritons in a phonon gas, $S_{\mathrm{ph}}$ is the entropy of the phonon gas, $n_{3}$ is the number density of impuritons, $\kappa_{3}$ is a partial coefficient of thermal conductivity, and $\varepsilon_{\mathrm{i}}=p_{\mathrm{i}}^{2} / 2 m_{\mathrm{i}}$ is the kinetic energy of impuritons. The 
time $\tau_{\mathrm{D}}^{(\mathrm{iph})}$ is defined by (26) where the subscripts $\alpha, \beta$ refer to $p h, i$, respectively. For nondegenerate mixtures one has

$$
\frac{2}{3} \frac{{ }_{1}\left\langle\varepsilon_{\mathrm{i}} \mid 1\right\rangle_{1}}{{ }_{1}\langle 1 \mid 1\rangle_{1}}=T
$$

Figure 1 shows the calculated (from equation (29)) and measured in ([6]) values of the effective coefficient of thermal conductivity for a mixture with the concentration $x=1.39 \cdot 10^{-4}$. The contribution of $\kappa_{3}$ is negligible. At this concentration $\tau_{\mathrm{D}}^{(\mathrm{iph})}$ turns out to be equal to $\tau_{D \text { min }}^{(\mathrm{iph})}$, which corresponds to fast relaxation in the phonon system of this mixture.

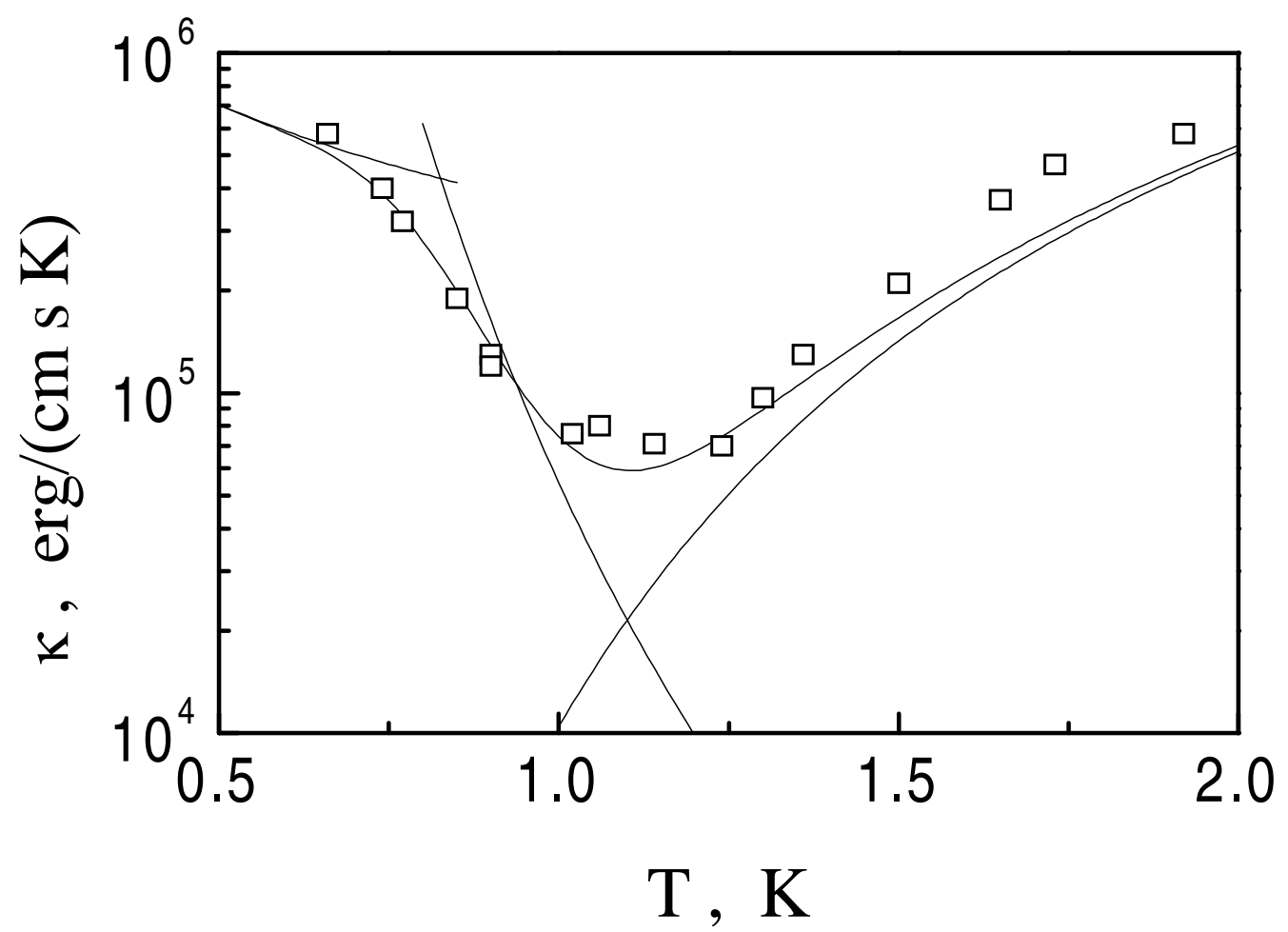

Figure 1. Temperature dependence of the effective thermal conductivity of a mixture with the concentration $x=1.39 \cdot 10^{-4}$, showing the contributions to the effective thermal conductivity from the diffusion in the impuriton-phonon (curve 1), roton-phonon (curve 2) and impuriton-roton (curve 3) systems. Curve 4 corresponds to the effective thermal conductivity calculated by taking into account contributions from all the quasiparticles. The experimental data obtained in [6] are represented by $\square$.

Figure 2 gives the observed ([6] and [7]) and calculated values of the effective coefficient of the thermal conductivity of a mixture with different concentrations. The figure shows that in the temperature region considered, with the increase in concentration the coefficient $\kappa_{\text {eff }}$ with $\tau_{\mathrm{D}}^{(\mathrm{iph})}=\tau_{D \text { min }}^{(\text {(iph }}$ (dashed lines) differs from the values obtained from formula (26), which takes into account the finite values of the phonon-phonon relaxation times. The values calculated by formula (26) 
are presented by solid curves and are in better agreement with the experimental data. The results for the considered temperature region correspond to the calculations of $[3,8,9]$. In the region of intermediate temperature $(0.7 \mathrm{~K}<T<1 \mathrm{~K})$, when the concentration of impuritons is small, the effective thermal conductivity is determined mainly by diffusion in the gas of thermal excitations.

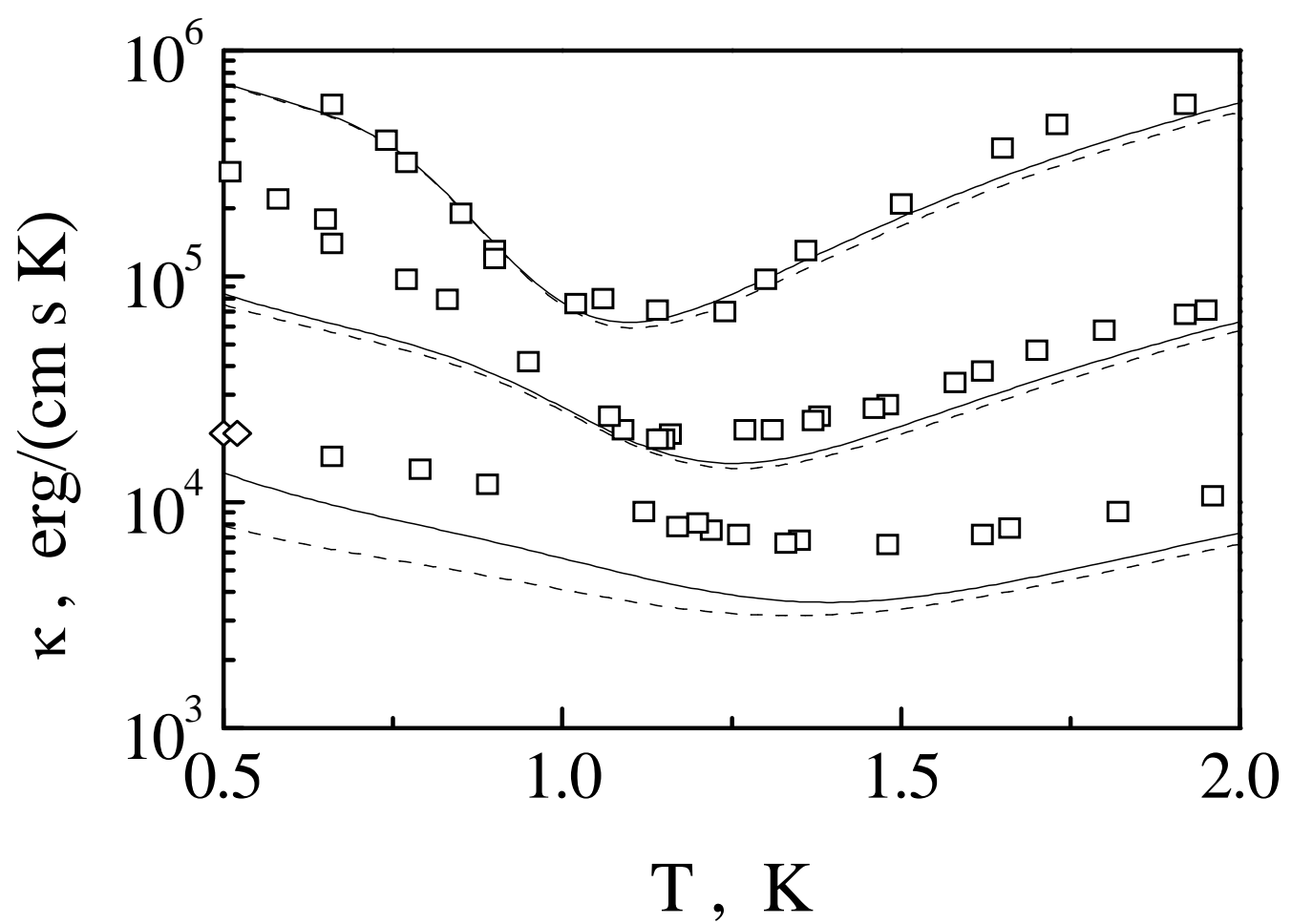

Figure 2. Temperature dependence of the effective thermal conductivity of the mixtures with different concentrations: $x=1.39 \cdot 10^{-4}$ (curve 1 ), $x=1.32 \cdot 10^{-3}$ (curve 2) and $x=1.36 \cdot 10^{-2}$ (curve 3 ). The results of the calculations taking into account the contributions from all the types of quasiparticles are depicted by solid and dashed curves. The latter correspond to calculations with the assumption of instantaneous relaxation in the mixture components. The experimental results obtained in [6] and [7] are represented by $\square$ and $\diamond$.

Diffusion processes in a phonon-roton gas were first considered in [10]. Later, in [1], these processes were shown to be the reason for a thermal transfer caused by a difference of dispersion laws of phonons and rotons. The calculation from relation (26) is analogous to that made in [1] and gives

$$
\kappa_{\mathrm{eff}} \approx \kappa_{\mathrm{D}}^{(\mathrm{rph})}=T^{-1} \frac{\rho_{\mathrm{ph}} \rho_{\mathrm{r}}}{\rho_{\mathrm{ph}}+\rho_{\mathrm{r}}}\left\{\frac{S_{\mathrm{ph}} T}{\rho_{\mathrm{ph}}}-\frac{S_{\mathrm{r}} T}{\rho_{\mathrm{r}}}\right\}^{2} \tau_{\mathrm{D}}^{(\mathrm{rph})} .
$$

Here $S_{\mathrm{r}}$ and $\rho_{\mathrm{r}}$ are the entropy and normal density of rotons, and $\tau_{\mathrm{D}}^{(\mathrm{rph})}$ is given by equation (26) in which the subscripts $\alpha, \beta$ should be substituted with $r, p h$, respectively. Expression (32) gives the result of [1] when the times $t_{\mathrm{phph}}$ and $t_{\mathrm{rr}}$ are equal to zero. Curve 3 in figure 1 presents the calculations from equation (32) and 
shows the existence of a wide enough temperature range, such that $\kappa_{\mathrm{D}}$ should be taken into account.

At high enough temperatures $(T>1 \mathrm{~K})$ the kinetic properties of superfluid mixtures are governed by rotons and impuritons. According to [11], in this temperature region, the coefficient of effective thermal conductivity can be written as

$$
\kappa_{\mathrm{eff}}=D_{\mathrm{ir}}\left(S_{\mathrm{r}} \frac{\rho_{\mathrm{r}}+\rho_{\mathrm{i}}}{\rho_{\mathrm{r}}}\right)^{2} n_{3}^{-1}+\kappa_{3}+\kappa_{\mathrm{r}}
$$

where $\kappa_{\mathrm{r}}$ is thermal conductivity of rotons.

Using the relations (1) and (26), the diffusion coefficient of impuritons in a roton gas is given as

$$
D_{\mathrm{ir}}=\frac{\rho_{\mathrm{r}}}{\rho_{\mathrm{r}}+\rho_{\mathrm{i}}} \frac{T}{m_{\mathrm{i}}} \tau_{\mathrm{D}}^{(\mathrm{ir})} .
$$

Here $\tau_{\mathrm{D}}^{\text {(ir) }}$ is given by relation (26), where the subscripts $\alpha, \beta$ should be substituted by $r, i$, respectively. The relation (34) gives the result of [1] if the times $t_{\mathrm{ii}}$ and $t_{\mathrm{rr}}$ are equal to zero. The rate of the roton-impuriton interaction can be written in the form [12]

$$
\begin{gathered}
t_{\mathrm{ir}}^{-1}\left(p_{\mathrm{i}}\right)=A^{2} n_{\mathrm{r}}\left(\int_{0}^{\infty} \exp \left\{-\frac{\mu v_{\mathrm{r}}^{2}}{2 T}\right\} \mathrm{d} v_{\mathrm{r}}\right)^{-1} \\
\times \int_{0}^{\infty} \exp \left\{-\frac{\mu v_{\mathrm{r}}^{2}}{2 T}\right\} \mathrm{d} v_{\mathrm{r}} \frac{1}{2} \int_{-1}^{1} \sin ^{2} \theta\left(\frac{\mu}{2}\left(\mathbf{v}_{\mathrm{r}}-\mathbf{v}_{\mathrm{i}}\right)^{2}+\frac{p_{\mathrm{i}}^{2}}{2 m_{\mathrm{i}}} \sin ^{2} \theta\right)^{\frac{1}{2}} \mathrm{~d}(\cos \theta)
\end{gathered}
$$

where $A$ is a scattering amplitude.

The limiting relations for the relaxation rates refer to the absence of equilibrium in the impuritons $\left(t_{\mathrm{ii}} \gg t_{\mathrm{ir}}\right)$ and fast relaxation in the roton gas $\left(t_{\mathrm{rr}} \ll t_{\mathrm{ri}}\right)$. Under these conditions the general expression (26) gives

$$
\tau_{\mathrm{D}}^{(\mathrm{ir})}=\tau_{\mathrm{D} \max }{ }^{(\mathrm{ir})}=\left\langle t_{\mathrm{ir}}\right\rangle_{\mathrm{i}} .
$$

The results calculated from equation (34) for the effective thermal conductivity for a mixture with $x=1 \cdot 10^{-4}$ are presented in figure 1 (curve 1 ). Here the contributions of the third and fourth terms in the right-hand side of equation (33) can be neglected. The account of the finite values of $t_{\mathrm{ii}}$ and $t_{\mathrm{rr}}$ makes the calculated values greater to the order of $10 \%$, improving the agreement between theory and experiment. In figure 2 , the dashed curves present calculations with $\tau_{\mathrm{D}}=\tau_{D \text { min }}$, and the solid curves correspond to $\tau_{\mathrm{D}}$ calculated from equation (26).

\section{Diffusion in solid ${ }^{3} \mathrm{He}-{ }^{4} \mathrm{He}$ mixtures}

The methods used in the previous section allow us to calculate the contribution of the phonon-impuriton interaction to spin diffusion of impurities in solid ${ }^{3} \mathrm{He}-{ }^{4} \mathrm{He}$ mixtures as

$$
D_{\mathrm{iph}}^{(\mathrm{s})}=\frac{2}{3} \frac{{ }_{1}\left\langle\varepsilon_{\mathrm{i}} \mid 1\right\rangle_{1}}{{ }_{1}\langle 1 \mid 1\rangle_{1}} \frac{1}{m_{\mathrm{i}}} \tau_{\text {phi }}^{(0)}
$$




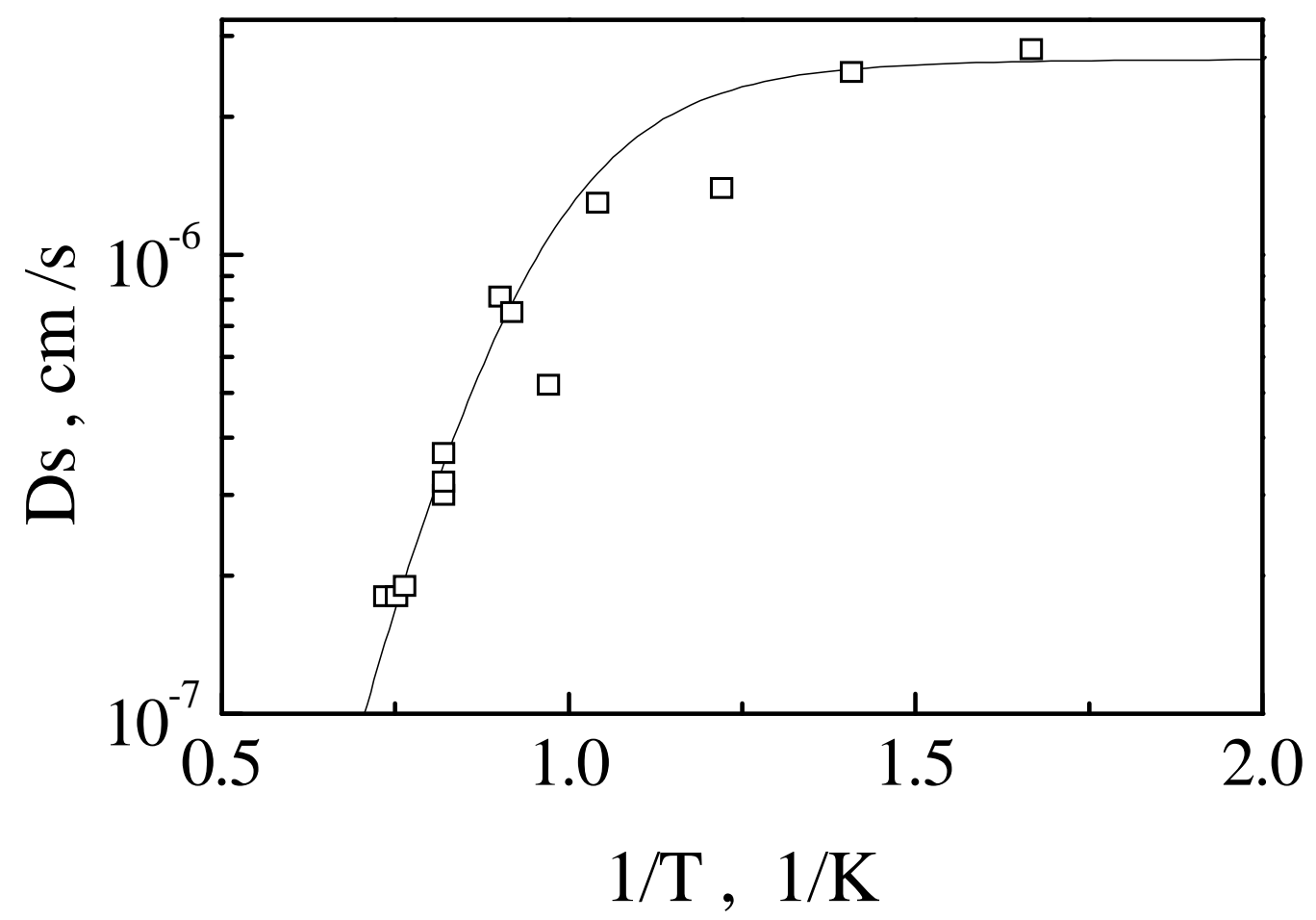

Figure 3. Dependence of the spin diffusion coefficient of solid ${ }^{3} \mathrm{He}-{ }^{4} \mathrm{He}$ mixtures on the reciprocal temperature for the concentration $x=6 \cdot 10^{-5}$. $\square$ stands for the experimental data of [20-22], and the curve corresponds to the calculations by equations (39-42).

Calculating the scalar products in (37), one should take integrals in the limit of the impurity energy band $\Delta \ll T$, thus giving

$$
D_{\mathrm{iph}}^{(\mathrm{s})}=\frac{2 \Delta}{5 m_{\mathrm{i}}} \tau_{\mathrm{iph}}^{(0)} .
$$

This result differs from the diffusion coefficient in the phonon-impuriton system of a liquid ${ }^{3} \mathrm{He}-{ }^{4} \mathrm{He}$ mixture, especially by its temperature dependence. To examine this, we rewrite equation (38) by using the definition (18):

$$
D_{\mathrm{iph}}^{(\mathrm{s})}=\frac{4}{25} \frac{\Delta^{2}}{T} \frac{n_{\mathrm{i}}}{\rho_{p h}} \tau_{\mathrm{phi}}^{(0)} .
$$

The time $\tau_{\text {phi }}^{(0)}$ has a typical Rayleigh scattering temperature dependence $T^{-4}$, and $\rho_{\text {ph }}$ is proportional to $T^{4}$, so that $D_{\mathrm{iph}}^{s} \sim T^{-9}$. Such a dependence was first obtained in [13] from phenomenological arguments. According to equation (38), this dependence is completely determined by $\tau_{\mathrm{iph}}^{(0)}$, and from equation (39) it follows that eight powers of temperature deal with phonons (normal density and scattering rate), and one power deals with the normal density of impuritons. The expressions (38) and (39) differ from the results of [13, 14-18]. Relation (39) includes parameters which can be directly determined from independent experiments on thermal 
conductivity in solid ${ }^{3} \mathrm{He}-{ }^{4} \mathrm{He}$ mixtures [19]. The numerical values of $\Delta$ can be obtained from the experimental data [20-22] for a mixture with $x=6 \times 10^{-5}$. The expression for spin diffusion can be written in the form

$$
D_{\mathrm{s}}=\left(D_{\mathrm{iph}}^{(\mathrm{s})^{-1}}+D_{\mathrm{ii}}^{-1}\right)^{-1}
$$

where $D_{\text {ii }}$ is independent of the temperature contribution of the impuriton- impuriton interaction to spin diffusion, which according to [23] is

$$
D_{\mathrm{ii}}=2.67 \cdot 10^{-7} \mathrm{~cm}^{2} / \mathrm{s} \text {. }
$$

Figure 3 presents the experimental [18] and calculated (by formula (41)) values of the diffusion coefficients. An agreement between the calculated and observed data is achieved with

$$
\Delta=3.5 \cdot 10^{-4} \mathrm{~K}
$$

The obtained value (42) refines the results of $[17,18]$ which give the order of magnitude only.

\section{References}

1. Adamenko I.N., Nemchenko K.E. Diffusion of quasiparticles of ${ }^{3} \mathrm{He}^{-}{ }^{4} \mathrm{He}$ mixtures and classical particles. // Fiz. Nizk. Temp., 1995, vol. 21, p. 498-508 (in Russian).

2. Fertziger J.H., Kaper H.G. Mathematical Theory of Transport Processes in Gases. Amsterdam, North-Holland, 1972.

3. Adamenko I.N., Rudavskii E.Ya., Tsyganok V.I., Chagovets V.K. New relaxation process in the phonon-impuriton system. // Pis'ma Zh. Eksp. Teor. Fiz., 1984, vol. 33, p. 404-407 (in Russian).

4. Callaway J. Model for lattice thermal conductivity at low temperatures. // Phys. Rev., 1959, vol. 113, p. 1046-1051.

5. Landau L.D., Pomeranchuk I.Ya. About the motion of outside particles in helium II. // Dokl. Ak. Nauk SSSR, 1948, vol. 59, p. 669-671 (in Russian).

6. Ptukha T.P. Thermal conductivity and diffusion of dilute ${ }^{3} \mathrm{He}-{ }^{4} \mathrm{He}$ solutions in the temperature range of from $\lambda$-point to 0.6 K. // Zh. Eksp. Teor. Fiz., 1961, vol. 40, p. 1583-1593 (in Russian).

7. Abel W.B., Wheatley J.C. Experimental thermal conductivity of two dilute solutions of ${ }^{3} \mathrm{He}$ in superfluid ${ }^{4}$ He. // Phys. Rev. Lett., 1968, vol. 21, p. 1231-1234.

8. Adamenko I.N., Tsyganok V.I. Change of phonon kinetics due to dispersion law and impurities. // Zh. Eksp. Teor. Phys., 1984, vol. 87, p. 865-877 (in Russian).

9. Adamenko I.N., Tsyganok V.I. Collective modes and dissipative coefficients in solutions of ${ }^{3} \mathrm{He}-{ }^{4} \mathrm{He}$ quantum liquids. // Zh. Eksp. Teor. Phys., 1985, vol. 88, p. 1641-1655 (in Russian).

10. Um C.I., Jun C., Shin H., George T.F. Quasi-diffusion between phonon and roton gases in two- and three-dimentional liquid helium. // J. Low Temp. Phys., 1990, vol. 78, p. $51-61$.

11. Khalatnikov I.M. The Theory of Superfluidity. Nauka, Moskow, 1971 (in Russian). 
12. Adamenko I.N., Zhukov A.V., Nemchenko K.E. Contribution of the interaction of identical particles to the diffusion of classical and quantum mixtures. // Fiz. Nizk. Temp., 1996, vol. 22, p. 1470-1473 (in Russian).

13. Andreev A.F., Lifshitz I.M. Quantum theory of defects in crystals. // Zh. Eksp. Teor. Fiz., 1969, vol. 56, p. 2057-2058 (in Russian).

14. Pushkarov I. To the theory of the motion of impurities in solid ${ }^{4} \mathrm{He}$. // Pis'ma Zh. Eksp. Teor. Fiz., 1974, vol. 19, p. 751-752 (in Russian).

15. Kagan Yu., Maksimov L.A. Theory of particle transport in extrimely narrow bands. // Zh. Eksp. Teor. Fiz., 1973, vol. 65, p. 622-639 (in Russian).

16. Kagan Yu., Klinger M.I. Theory of quantum diffusion of atoms in crystals. // J. Phys. C 1974, vol. 7, p. 2791-2808.

17. Slusarev V.A., Strzhemechnyi M.A., Burachovich I.A. Quantum diffusion of ${ }^{3} \mathrm{He}$ in solid ${ }^{4}$ He. I. The role of impurity interaction anisotropy. // Fiz. Nizk. Temp., 1977, vol. 3, p. 1229-1240 (in Russian).

18. Slusarev V.A., Strzhemechnyi M.A., Burachovich I.A. Quantum diffusion of ${ }^{3} \mathrm{He}$ in solid ${ }^{4}$ He. II. Tunneling-jumping approximation. // Fiz. Nizk. Temp., 1978, vol. 4, p. 698-705 (in Russian).

19. Golub A.A., Svatko S.V. Density dependence of the thermal conductivity of $\mathrm{HCP}^{3} \mathrm{He}$ in ${ }^{4}$ He solutions. // Fiz. Nizk. Temp., 1981, vol. 7, p. 970-976 (in Russian).

20. Grigor'ev V.N., Esel'son B.N., Mikheev V.A. New peculiarities of quantum diffusion of ${ }^{3} \mathrm{He}$ in solid helium. // Pis'ma Zh. Eksp. Teor. Fiz., 1973, vol. 18, p. 289-291 (in Russian).

21. Grigor'ev V.N., Esel'son B.N., Mikheev V.A., et al. ${ }^{3}$ He impurity excitations in solid ${ }^{4}$ He. // J. Low Temp. Phys., 1973, vol. 13, p. 65-86.

22. Grigor'ev V.N, Esel'son B.N., Mikheev V.A. Diffusion of ${ }^{3} \mathrm{He}$ in BCC- and HCP-phases of solid solutions of helium isotopes. // Zh. Eksp. Teor. Fiz., 1974, vol. 66, p. 321-329 (in Russian).

23. Esel'son B.N., Grigor'ev V.N., Ivonsov V.G, et al. Solutions of Quantum Liquids ${ }^{3}$ He${ }^{4}$ He. Nauka, Moscow, 1973 (in Russian). 


\title{
Дифузія у двокомпонентних системах квазічастинок рідких та твердих сумішей ізотопів гелію
}

\author{
І.Н.Адаменко ${ }^{1}$, К.Е.Немченко ${ }^{1}$, А.В.Жуков ${ }^{1}$, \\ Томас Ф. Джордж ${ }^{2}$, Лакшмі Н. Пенді ${ }^{3}$, Чанґ-Ін Ум ${ }^{4}$ \\ 1 Харківський державний університет, \\ 310077 Харків, пл. Свободи, 4 \\ 2 Університет Вісконсіна - Стівенс Пойнт, \\ факультети хімії, фізики і астрономії, Вісконсін 54481-3897, США \\ 3 Університет штату Вашінгтон, факультети хімії і фізики, \\ Пуллмен, Вашінгтон 99164-4630, США \\ 4 Університет Кореї, факультет фізики, коледж природничих наук, \\ Сеул 136-701, Корея
}

\section{Отримано 5 грудня 1997 р.}

Виходячи з системи кінетичних рівнянь для компонент суміші отримано точний вираз для дифузійного часу, що залежить від взаємодії частинок не тільки різних, а й одного типу. Отриманий результ справедливий для частинок з довільною дисперсією і статистикою. Одержаний точний вираз використовується для дослідження дифузії у рідких та твердих квантових розчинах ${ }^{3} \mathrm{He}-{ }^{4} \mathrm{He}$. Проаналізовано внесок взаємодії між квазічастинками однакових типів у коефіцієнт дифузії та теплопровідності надплинних розчинів. Обчислені результати порівнюються з існуючими експериментальними даними. Отриманий коефіцієнт дифузії для твердих квантових розчинів ${ }^{3} \mathrm{He}-{ }^{4} \mathrm{He}$ істотно відрізняється від попередніх результатів теорії. Порівняння цього результату з експериментальними даними дозволило дістати числове значення для енергетичної зони квазічастинок домішки.

Ключові слова: дифузія, теплопровідність, гелій, квантова рідина,

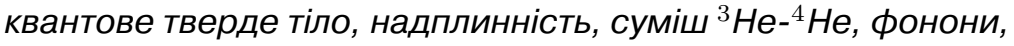
ротони, домішки.

PACS: $51.20 .+d, 67.80 . \mathrm{Mg}$ 